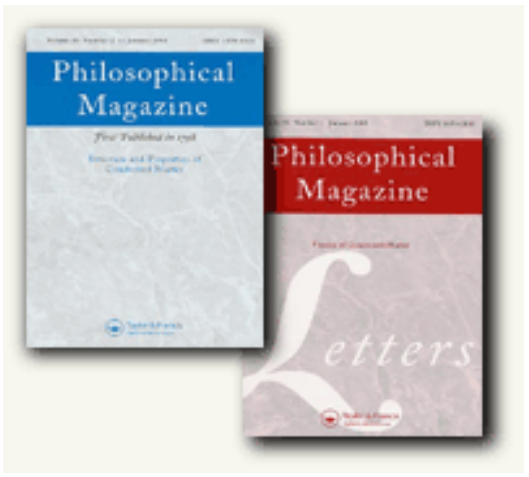

\title{
A three-dimensional continuum theory of dislocation systems: Kinematics and mean-field formulation
}

\begin{tabular}{|r|l|}
\hline Journal: & Philosophical Magazine \& Philosophical Magazine Letters \\
\hline Manuscript ID: & TPHM-06-Mar-0055.R1 \\
\hline Authal Selection: & Philosophical Magazine \\
\hline Complete List of Authors: & $\begin{array}{l}\text { Hochrainer, Thomas; Universitaet Karlsruhe (TH), Institut fuer } \\
\text { Zuverlaessigkeit von Bauteilen und Systemen } \\
\text { Zaiser, Michael; The University of Edinburgh, King's Buildings, } \\
\text { Sanderson Building, Center for Materials Science and Engineering } \\
\text { Gumbsch, Peter; Universitaet Karlsruhe (TH), Institut fuer } \\
\text { Zuverlaessigkeit von Bauteilen und Systemen; Fraunhofer Institut } \\
\text { fuer Werkstoffmechanik IWM }\end{array}$ \\
\hline Keywords: & dislocation mechanics, multiscale modelling \\
\hline Keywords (user supplied): & dislocation densities, conservation laws, mean field theory \\
\hline & \\
\hline $\begin{array}{l}\text { Note: The following files were submitted by the author for peer review, but cannot be converted } \\
\text { to PDF. You must view these files (e.g. movies) online. }\end{array}$ \\
\hline TPHM-06-Mar-0055_Hochrainer.tex
\end{tabular}

\section{S ScholaroNE \\ Manuscript Central}




\title{
A three-dimensional continuum theory of dislocation systems: Kinematics and mean-field formulation
}

\author{
T. HOCHRAINER ${ }^{*} \dagger$, M. ZAISER $\ddagger$, P. GUMBSCH $\dagger \S$
}

$\dagger$ †niversität Karlsruhe (TH), Institut für Zuverlässigkeit von Bauteilen und Systemen, Kaiserstr. 12, 76131 Karlsruhe, Germany

$\ddagger$ The University of Edinburgh, Centre for Materials Science and Engineering, King's Buildings, Sanderson Building, Edinburgh EH93JL, United Kingdom

${ }^{\S}$ Fraunhofer-Institut für Werkstoffmechanik IWM, Wöhlerstr. 11, 79108 Freiburg, Germany

\begin{abstract}
We propose a dislocation density measure which is able to account for the evolution of systems of three-dimensionally curved dislocations. The definition and evolution equation of this measure arise as direct generalisations of the definition and kinematic evolution equation of the classical dislocation density tensor. The evolution of this measure allows to determine the plastic distortion rate in a natural fashion and therefore yields a kinematically closed dislocation-based theory of plasticity. A self-consistent theory is built upon the measure which accounts for both the long range interactions of dislocations and their short range self-interaction which is incorporated via a line tension approximation. A two-dimensional kinematic example visualises the definitions and their relations to the classical theory.
\end{abstract}

Keywords: Dislocation densities; Conservation Laws; Mean field theory

\section{Introduction}

It is a central aim of current materials modelling to build effective continuum theories directly on averages of the corresponding discrete objects. In the case of crystal plasticity the most important objects to be looked at are dislocations and their dynamics. Recently averaging procedures adapted from the statistical mechanics of interacting many-particle systems have successfully been used to deduce a continuum description for simplified dislocation systems consisting of straight parallel edge dislocations of both signs and moving on a single slip system [1]. This corresponds to dealing with the dynamics of signed point particles moving in a plane. The evolution equations for the corresponding densities

\footnotetext{
*Corresponding author. Email: thomas.hochrainer@izbs.uka.de
} 
$\rho_{ \pm}$therefore have the form $\partial_{t} \rho_{ \pm}=-\operatorname{div}\left(\rho_{ \pm} \boldsymbol{v}_{ \pm}\right)$with $\boldsymbol{v}_{ \pm}$denoting the dislocation velocity. These kinematic evolution equations, which in the present form have the structure of conservation laws, are in fact the fundament upon which the statistical mechanics methods are built ${ }^{\top}$. Consequently, in order to transfer the statistical mechanics approach to three dimensional curved dislocations, the definition of a mathematically sound dislocation density measure and the formulation of its kinematic evolution law are essential.

The classical dislocation density tensor $\boldsymbol{\alpha}$ as introduced by Kröner [2] constitutes a three-dimensional dislocation density measure which considers the line like character of the dislocations. In fact it yields a proper pseudo continuum description for single dislocations and a continuum description of special dislocation distributions. However, it is well known that upon averaging $\boldsymbol{\alpha}$ often only reflects a small fraction of all dislocations and that therefore no closed plasticity theory can be built upon it. Indeed, already the above mentioned 2D theory exceeds the scope of the dislocation density tensor which would in that case correspond to the net-density $\kappa=\rho_{+}-\rho_{-}$. It is obvious that for a closed description which is equivalent to the evolution of the two 'signed' densities the evolution equation for $\kappa$ has to be accompanied by a second equation for the total dislocation density $\rho=\rho_{+}+\rho_{-}$.

A rather natural generalisation of the concept of two signed densities (for straight parallel dislocations) to more general dislocation configurations is to introduce a continuous directional space and a density function $\rho$ on the sphere of directions at each point of the crystal. This concept goes back to Kosevich [3] and also yields the back-bone for the statistical mechanics theory of El-Azab [4], who additionally classifies dislocations by their velocity. It was shown in [5] that the associated dislocation density measures (in the following denoted as 'statistical' measures; the motivation for this terminology will become apparent later) and their generalisations can yield a closed plasticity theory only in very special situations.

In [6] we proposed a different, 'deterministic' generalisation of the classical dislocation density tensor which is akin to the above mentioned statistical ones but additionally considers the local average curvature of dislocations of a fixed orientation. The main purpose of the current paper is to motivate the definition and evolution equation of this dislocation density measure, and to relate them to the classical theory and the cited statistical approaches. We will discuss why the associated evolution equation constitutes an appropriate 'continuity equation' (conservation law) for curved lines. As a first step towards a dynamic theory we will show how a fully three-dimensional self-consistent (or mean field) theory of plasticity may be built upon the presented dislocation density measure. Finally we give a detailed two-dimensional calculation to illustrate the definitions and equations. A simple example problem is analysed, but we refer the reader to [7] (this volume) for an application of the present theory to size-effects in thin film plasticity.

A mathematically sound definition of the generalised dislocation density measure requires to use the language of differential forms because higher dimen-

IOnce a kinematic evolution equation is established, the crucial physical problem in statistical modelling is to determine the velocity $\boldsymbol{v}$ and establish its relation with the dislocation densities. In the cited case it turns out that, under the assumption of overdamped dislocation motion, the velocity can be written as a functional of the dislocation densities which encompasses several stress contributions accounting for different kinds of dislocation interactions. 
sional generalisations of the cross product $x$ and the curl-operator are needed (see [6] for a discussion of other motivations for the use of differential forms in the present context). Consequently we will use the language of differential forms throughout the whole paper, including the presentation of the classical theory, but include for comparison the standard formulation where appropriate. As the definitions and notations used are not widely known outside the differential geometry community, we give a collection of definitions and standard results in Appendix A. At the beginning of Appendix A we also discuss the understanding of tangent vectors as differential operators acting on functions and introduce the corresponding concepts, definitions and notations. However, we avoid in this work the use of more elaborate concepts from differential geometry which are in fact needed to formulate some of the results in a more precise and general fashion, and refer the interested reader to [5] for a rigorous treatment. Instead we rely, where ever possible, on geometrical and physical arguments to motivate the use of a certain mathematical formulations and to explain the implications of apparently formal mathematical choices.

\section{Current dislocation density measures}

In this section we first give a detailed review of the classical dislocation density tensor and translate the classical results into the language of differential forms. Afterwards we briefly discuss statistical dislocation density measures found in the literature before we introduce the generalised deterministic dislocation density measure and its evolution equation. We will then recover the classical theory from the generalised one and finally interpret the evolution equation as a conservation law.

\subsection{The classical dislocation density tensor}

The classical dislocation density tensor was introduced independently and with slightly different accents by Nye [8] and Kröner [2] as well as in a much more formal way by Kondo [9] and Bilby et al. [10]. In the following we use the definitions and notations by Kröner [11].

It was set out in [6] why the classical dislocation density tensor $\boldsymbol{\alpha}$ naturally arises as a vector valued 2-form. This was already appreciated by Kröner [11]. Considering $\boldsymbol{\alpha}$ as a 2 -form means that we prefer to work with the third rank tensor"l

$$
\boldsymbol{\alpha}=\alpha_{i j}{ }^{k} d x^{i} \wedge d x^{j} \otimes \partial_{k},
$$

which is antisymmetric in the two lower indices, instead of the more commonly used rank two tensor

$$
\boldsymbol{\alpha}=\alpha^{i j} \partial_{i} \otimes \partial_{j} .
$$

Both tensors are related via the totally antisymmetric symbols $\epsilon_{i j k}$ and $\epsilon^{i j k}$ by

$$
\alpha_{i j}^{k}=\epsilon_{l i j} \alpha^{l k} \quad \text { and } \quad \alpha^{i j}=\epsilon^{i k l} \alpha_{k l}^{j} .
$$

As usual in the theory of Kröner we transfer differential operators from vector calculus to tensor calculus by applying them only to specific indices of a tensor in

\footnotetext{
${ }$ Here and in the following we adopt the Einstein summation convention for automatically contracting over pairs of upper and lower indices.
} 
Cartesian coordinates. For example the divergence of $\boldsymbol{\alpha}$ is traditionally defined by

$$
\operatorname{div} \boldsymbol{\alpha}:=\partial_{i} \alpha^{i j} \partial_{j}
$$

Analogously the usual calculus of differential forms can be transferred to vectorvalued differential forms by applying them to the 'lower' indices as for example

$$
d \boldsymbol{\alpha}=d \alpha_{i j}{ }^{k} \wedge d x^{i} \wedge d x^{j} \otimes \partial_{k},
$$

which is the equivalent to equation (1). We remark that both definitions in this form rely on the use of standard coordinates.

Analogous to the understanding of $\boldsymbol{\alpha}$ as a vector-valued 2-form, the plastic distortion tensor $\boldsymbol{\beta}^{\mathrm{pl}}$ shall be viewed as a vector-valued 1-form $\boldsymbol{\beta}^{\mathrm{pl}}=\beta^{\mathrm{pl}}{ }_{i}^{j} d x^{i} \otimes$ $\partial_{j}$. The classical definition of the dislocation density as curl of the plastic distortion then reads in the notion of differential forms

$$
\boldsymbol{\alpha}=\operatorname{curl} \boldsymbol{\beta}^{\mathrm{pl}} \longleftrightarrow \quad \boldsymbol{\alpha}=d \boldsymbol{\beta}^{\mathrm{pl}} .
$$

Similarly the fact that dislocations do not end inside the crystal is rewritten as

$$
\operatorname{div} \boldsymbol{\alpha}=0 \longleftrightarrow d \boldsymbol{\alpha}=0 .
$$

We remark that the conceptual difference between the vector-valued 1-form $\boldsymbol{\beta}^{\mathrm{pl}}$ and the vector-valued 2-form $\boldsymbol{\alpha}$ which does not clearly show up in the traditional notation can be motivated from the fact that $\boldsymbol{\beta}^{\mathrm{pl}}$ is meaningfully integrated over curves (to yield the enclosed Burgers vector) while $\boldsymbol{\alpha}$ can only be meaningfully integrated over surfaces (to yield the net Burgers vector flux through the surface). It was shown in [6] using the concept of currents that this can be related to the fact that $\boldsymbol{\beta}^{\mathrm{pl}}$ may be seen as representing a density of plastically sheared surfaces (where the direction and amount of slip is given by the Burgers vector), while $\boldsymbol{\alpha}$ represents a density of curves. It was furthermore shown that equation (2) literally reflects the fact that dislocation lines emerge as the boundaries of slipped surfaces, and that equation (3) is the continuum version of dislocation lines being closed.

In the remainder of this paper, we for simplicity assume all dislocations to have the same Burgers vector $\boldsymbol{b}$. As a consequence, the vectorial part of the vector-valued differential forms is constant and given by the Burgers vector. Therefore the calculus effectively reduces to the usual calculus of differential forms and the treatment of dislocations reduces to a theory of distributed curves. Consequently the dislocation density tensor can be written as

$$
\boldsymbol{\alpha}=\rho \mathrm{i}_{l} d V \otimes \boldsymbol{b},
$$

with a density function $\rho$ and a unit vector field $\boldsymbol{l}$ which gives the local average direction of the dislocation lines. Furthermore $d V$ denotes the standard volume element and ' $\mathrm{i}$ ' is the operator of inner multiplication of a differential form with a vector field, as introduced in Appendix A. In a standard Cartesian co-ordinate system in three dimensions this reads

$$
\boldsymbol{\alpha}=\rho\left(l^{1} d x^{2} \wedge d x^{3}-l^{2} d x^{1} \wedge d x^{3}+l^{3} d x^{1} \wedge d x^{2}\right) \otimes \boldsymbol{b}^{i} \partial_{i}
$$

It is well known that the classical dislocation density tensor does - as an averaged object - not carry enough information about the dislocation state to 
deduce the rate of plastic deformation $\partial_{t} \boldsymbol{\beta}^{\mathrm{pl}}$ from it. As $\partial_{t} \boldsymbol{\alpha}=\operatorname{curl} \partial_{t} \boldsymbol{\beta}^{\mathrm{pl}}$, or equivalently in the notion of differential forms $\partial_{t} \boldsymbol{\alpha}=d \partial_{t} \boldsymbol{\beta}^{\mathrm{pl}}$, the absence of a relation between $\boldsymbol{\alpha}$ and the deformation rate implies that the evolution of $\boldsymbol{\alpha}$ itself cannot be formulated in closed form. In other words it is in general not possible to build a closed theory of plasticity solely on the classical dislocation density tensor. However, the classical formulation is suited for the treatment of single dislocations as singular densities, as well as for the special situations where dislocations form smooth line bundles (dislocations in the same volume element are parallel and have the same orientation), which is e.g. exploited by Sedláček et al. in [12]. Only in these cases the density function $\rho$ coincides with the total dislocation density and the average line-direction $\boldsymbol{l}$ actually gives the true direction of the dislocation lines. Because of the latter fact it makes in this case sense to assign to the dislocation density a smooth velocity field $\boldsymbol{v}$, which is at each point orthogonal to the line direction $\boldsymbol{l}$ of the dislocations. The plastic distortion tensor can consequently be deduced from Orowan's equation as $\partial_{t} \boldsymbol{\beta}^{\mathrm{pl}}=\rho v \boldsymbol{n} \otimes \boldsymbol{b}$ in traditional notation for conservative glide. Here $v$ denotes a pseudo scalar velocity and $\boldsymbol{n}$ the glide plane normal. The differential form version of this looks virtually the same if we introduce the dual 1-form $\boldsymbol{n}^{\mathrm{b}}$ to $\boldsymbol{n}$. This is defined by its action on a vector $\boldsymbol{x}$ by assigning the scalar product with $\boldsymbol{n}$, thus $\boldsymbol{n}^{b}(\boldsymbol{x})=\boldsymbol{n} \cdot \boldsymbol{x}$. The plastic distortion rate as differential form then reads

$$
\partial_{t} \boldsymbol{\beta}^{\mathrm{pl}}=\rho v \boldsymbol{n}^{\mathrm{b}} \otimes \boldsymbol{b} .
$$

The corresponding closed evolution equation was first deduced by Mura [13]. We again compare the traditional notation to the differential form formulation to find

$$
\partial_{t} \boldsymbol{\alpha}=-\operatorname{curl}(\boldsymbol{v} \times \boldsymbol{\alpha}) \longleftrightarrow \partial_{t} \boldsymbol{\alpha}=-\mathcal{L}_{\boldsymbol{v}} \boldsymbol{\alpha},
$$

with $\mathcal{L}$ denoting the Lie-derivative introduced in Appendix A. It should be noted that the velocity field may in this case originate from virtually any law able to assign a velocity to a dislocation segment at any point.

In conclusion of our revision of the classical dislocation density measure, we discuss its evolution equation as given in the form deduced by Sedláček et al. [12] for a single glide system. The special form of this equation fosters the geometrical understanding of the evolution and will later serve as a reference for the explicit calculation of the generalised evolution equation in section 6 .

A family of dislocations with the same Burgers vector which are bound to move conservatively within the glide plane is considered. The glide plane can be parametrised with the Cartesian co-ordinates $x$ and $y$. The line-direction field $\boldsymbol{l}=l^{x} \partial_{x}+l^{y} \partial_{y}$ has unit length $\|\boldsymbol{l}\|=1$ with respect to the standard Euclidean norm. A velocity field $\boldsymbol{v}=v^{x} \partial_{x}+v^{y} \partial_{y}=v l^{y} \partial_{x}-v l^{x} \partial_{y}$ is given that is always orthogonal to $\boldsymbol{l}$. Again $v$ denotes a pseudo scalar velocity. Sedláček et al. use an illuminative notation in that they parametrise the line direction by an angle $\varphi$, thus $\boldsymbol{l}=\cos \varphi \partial_{x}+\sin \varphi \partial_{y}$, and consider $\varphi$ as a field variable. In this notation the evolution equation for $\boldsymbol{\alpha}=\rho \mathrm{i}_{l} d V \otimes \boldsymbol{b}$ can be split into an evolution equation for $\rho$ and one for $\varphi$ which are found as

$$
\partial_{t} \rho=-\operatorname{div}(\rho \boldsymbol{v})+\rho v k \quad \text { and } \quad \partial_{t} \varphi=\boldsymbol{l}(v)-v \operatorname{div} \boldsymbol{l},
$$

where $k$ denotes the curvature of the integral curves of $\boldsymbol{l}$ and hence of the dislocations. Here $l(v)$ denotes the application of a vector field to a 
(pseudo) scalar by acting as directional derivative, as discussed in Appendix A. We emphasise that the appearance of the curvature in a sort of source term $\rho v k$ for the density $\rho$ reflects the change of total line length in the system due to the expansion or shrinkage of curved dislocations.

\subsection{Advanced dislocation density measures}

Due to the fact that the classical dislocation density tensor in general gives only a very incomplete picture of a dislocation distribution, several more refined dislocation density measures have been proposed. A very appealing approach was proposed by El-Azab [4], who took up a concept first introduced by Kosevich [3], classifying dislocations at each point by their line direction**. This yields a measure $\boldsymbol{\alpha}_{\mathrm{e}}(r, \omega)=\rho(r, \omega) d V d \Omega$, where $\rho$ reflects the number of dislocations in a volume element $d V$ around the point $r$ with line direction contained in the solid angle element $d \Omega$ around the direction $\omega$. In accordance with the notation introduced for a class of similar measures in [5] we call this measure a statistical one. The term statistical refers to the fact that this measure gives only an incomplete picture of the dislocation state in the sense that after averaging essential information required for reconstructing the actual dislocation lines is lost (except for the case when one is dealing with distributions of only straight dislocations). It was pointed out earlier, see e.g. [14], that a draw-back of this definition is the fact that dislocation populations of different curvatures may, after averaging, be described by the same dislocation density. Consequently any evolution equation solely built on this measure can not account for the change in total dislocation line length due to expansion or shrinkage of dislocation loops which is relying on the curvature. From a formal point of view the measure furthermore suffers from the fact that the dislocation direction does not appear as a vector quantity but solely as a part of the configuration space. This would be needed to turn the spatial 3-form $d V$ into a 2 -form by inner multiplication. As shown in [5], this technical problem can be resolved by using the formal definition $\boldsymbol{\alpha}_{\mathrm{e}, \text { alt }}(p, \omega)=\rho(r, \omega) \mathrm{i}_{\boldsymbol{l}(\omega)} d V d \Omega$, which leaves the measure a statistical one. However, this formal modification cannot overcome the deficiency explained above.

The idea of a statistical dislocation density measure can be extended to descriptions of higher order [5], for example by including the dislocation curvature $\kappa$ into the configuration space. In the language of differential forms this yields the measure

$$
\boldsymbol{\alpha}_{\mathrm{s}}(r, \omega, \kappa)=\rho(r, \omega, \kappa) \mathrm{i}_{(\boldsymbol{l}(\omega), \boldsymbol{k}(\kappa), 0)} d V d \Omega d K \otimes \boldsymbol{b},
$$

where $\boldsymbol{k}=\boldsymbol{k}(\kappa)$ is the curvature vector corresponding to the curvature $\kappa$ and $d K$ denotes the 'volume element' in curvature space. We refer to the next section for more on the interpretation of the composed vector $(l, k, 0)$ as a higher order tangent to the dislocations. This statistical measure allows to reconstruct the dislocation lines only if all dislocations form circular loops or straight lines (loops with infinite radius). Nevertheless it could be

\footnotetext{
**El-Azab additionally classifies the dislocations by their velocity. If the dislocation velocity can be formulated as a function of the orientation and position in space of a dislocation, this classification is redundant (it is needed, on the other hand, if dislocations possess inertia). We will not consider the velocity-dependent classification within the present paper, as it complicates the presentation without helping to clarify the basic geometrical ideas.
} 
shown in [5] that the evolution of a dislocation configuration may be described by this measure if the dislocations are restricted to conservative motion within their glide plane and if the absolute value of the velocity of a dislocation segment only depends on the spatial point and not on the line direction or the curvature of the segment. These are still serious restrictions as compared to more realistic dislocation velocity laws (for instance segments of screw and edge orientation may have very different mobilities) and it was shown in [5] that even statistical approaches of higher order would not resolve this problem.

\section{The new measure and its relation to the clas- sical theory}

As opposed to the statistical dislocation density measures discussed above, we may denote the classical dislocation density tensor as a deterministic measure. This notation results from the fact that from this tensor the dislocation lines can be reconstructed as the flow lines (integral curves) of a vector field as long as the dislocations form smooth line bundles. In fact, both 'statistical' and 'deterministic' measures imply restrictions on the dislocation distributions for which a reconstruction of the dislocation lines is possible; the classical dislocation density tensor needs the assumption that dislocations form smooth line-bundles for which the orientation is unique at each point in space, whereas for the measure introduced by El-Azab they may have different orientations at a single point but the dislocation lines may be reconstructed only if the dislocations are straight. These restrictions are, however, of very different character. In particular, the restriction implicit in the deterministic measure will be conserved during the evolution independently of the nature of the applied velocity field, whereas this is not the case for the statistical measure as initially straight dislocations may bend in inhomogeneous velocity fields.

The advantages of both measures, i.e. the absence of constraints on the applicable velocity fields on the one hand, and the possibility of describing dislocation arrangements with dislocations of different directions in the same spatial volume element on the other hand, can be combined in a higher dimensional deterministic approach. This yields an object defined on the same configuration space as the measure defined by El-Azab, i.e. a space which is made up of points $r$, and of all directions $\omega$ at each point; this configuration space will be denoted with $S M$ in the following. If we denote the crystal manifold, i.e. the set of spatial points $r$, with $M$ and the unit sphere in three dimensions with $S^{2}$ we obtain $S M=M \times S^{2}$. Before we give the precise definition of the measure we shall motivate it from the analogy to the classical theory.

For the classical case to be exact we assumed that dislocations are distributed such that nearby dislocations have the same direction and orientation. This makes it possible to describe the dislocation state via a vector field $\boldsymbol{l}$. Thus $\rho(r)$ in this case gives the total density of dislocations (necessarily with the corresponding line direction $\boldsymbol{l}(r)$ ) at a certain point $r$. The integral curves $c$ of the vector field $\boldsymbol{l}$ correspond to the dislocation lines. If we take the curves to be parametrised by the arc-length $s$ and denote differentiation with respect to $s$ by a dot, we have $\dot{c}=\boldsymbol{l}$ along each curve. In the yet to be defined deterministic measure we do not consider as constituting objects the dislocation 
Figure 1: Visualisation of the lift of a moving dislocation. The red curve represents a circular dislocation loop which expands with the angular dependent velocity field depicted by black arrows orthogonal to the curve. The blue curve is the lift of the dislocation with the vertical axis giving the angle between the local line-direction and a fixed axis. Notice that the configuration space is periodic in the vertical direction and hence that the lifted curve is closed. The green arrows indicate the lifted velocity field. It should be noted that the vertical component of the tangent to the lifted curve corresponds to the curvature of the base curve and that similarly the rotational part $\boldsymbol{\vartheta}$ of the lifted velocity shows up in the vertical component of the latter.

lines themselves but rather their so-called 'lift' to the configuration space $S M$. The lift of a curve is defined via its tangent vector-field $\dot{c}$. If $c:[0, L) \rightarrow M$ is a parametrisation of a dislocation line by the arc-length $s$, then $\dot{c}$ is a unit vector-field along the curve. For each $s$ we can therefore assign a direction $\omega(s)$ which corresponds to $\dot{c}(s)$. Therefore the map

$$
\begin{aligned}
C:[0, L) & \rightarrow S M \\
s & \mapsto(c(s), \dot{c}(s))
\end{aligned}
$$

defines a curve in $S M$ which is called the lift of $c$. As an example the lift of a planar curve is shown in figure 1. Dislocations crossing the same spatial point in different directions may as lifts be clearly distinguished because they are going through different points in $S M$. For generalisation of the vector-field $\boldsymbol{l}$ we take a look at the tangent of $C$. The tangent is found as $\dot{C}(s)=(\dot{c}(s), \ddot{c}(s))$ and is a tangent vector to $S M$ at the point $C(s)=(c(s), \dot{c}(s))$. The tangent in its first part $\dot{c}$ coincides with the point in $S M$ while the second part $\ddot{c}$ is the curvature vector $\boldsymbol{k}$ of the curve. As a consequence, a vector-field $\boldsymbol{L}$ on $S M$ 
which corresponds to tangents of lifted curves must at the point $(r, \omega)$ have the form $\boldsymbol{L}(r, \omega)=(\boldsymbol{l}(\omega), \boldsymbol{k}(r, \omega))$ where the first part, which is the spatial linedirection, is determined by the considered point in $S M$. The curvature vector $\boldsymbol{k}$ must be orthogonal to $\boldsymbol{l}$ because the same holds for $\ddot{c}$ with respect to $\dot{c}$.

Considering this motivation we define a generalised 'deterministic' dislocation density measure as

$$
\boldsymbol{\alpha}_{\mathrm{d}}=\rho(r, \omega) \mathrm{i}_{(\boldsymbol{l}(\omega), \boldsymbol{k}(r, \omega))} d V d \Omega \otimes \boldsymbol{b},
$$

where $\boldsymbol{k}(r, \omega)$ is a curvature vector field which must at each orientation $\omega$ be orthogonal to $\boldsymbol{l}(\omega)$. If we introduce the volume element on $S M$ as $d \mathcal{V}=d V d \Omega$ the definition may be rewritten as

$$
\boldsymbol{\alpha}_{\mathrm{d}}=\rho \mathrm{i}_{\boldsymbol{L}} d \mathcal{V} \otimes \boldsymbol{b},
$$

which clearly shows the analogy to the classical definition via $\boldsymbol{\alpha}=\rho \mathrm{i}_{l} d V \otimes \boldsymbol{b}$.

Also the fact that dislocations do not end inside a crystal displays in full analogy to the classical theory in the requirement that $\boldsymbol{\alpha}_{\mathrm{d}}$ is closed, thus $d \boldsymbol{\alpha}_{\mathrm{d}}=0$ (lifts of closed curves are closed). This results in a coupling between the density function $\rho$ and the curvature vector field $\boldsymbol{k}$ because

$$
d \boldsymbol{\alpha}_{\mathrm{d}}=\operatorname{Div}(\rho \boldsymbol{L}) d \mathcal{V} \otimes \boldsymbol{b}=(\boldsymbol{L}(\rho)+\rho \operatorname{Div} \boldsymbol{L}) d \mathcal{V} \otimes \boldsymbol{b} .
$$

Here we denote with Div the divergence operator on the configuration space $S M$.

The analogy to the classical case can also be extended to the evolution equation. To this end we first have to define a generalised velocity $\boldsymbol{V}=(\boldsymbol{v}, \boldsymbol{\vartheta})$ which is capable of describing the motion of a lifted curve rather than just of the curve itself. If the motion of a dislocation line $c$ is given by a velocity $\boldsymbol{v}$ along the line, we find that the velocity of the lifted curve must additionally account for the rotation velocity $\boldsymbol{\vartheta}$ of the line-direction $\boldsymbol{\vartheta}:=\partial_{t} \boldsymbol{l}=\partial_{t} \frac{\dot{c}}{\|\dot{c}\|}$. See figure 1 for a visualisation of the concept. It is evident and can also be formally shown [5] that the change of the local line direction during the motion of a dislocation is determined by the derivative of the velocity along the dislocation line - more precisely, that part of the derivative which is orthogonal to the line direction. On the configuration space $S M$ the dislocation line is characterised by the generalised line-direction $\boldsymbol{L}(r, \omega)=(\boldsymbol{l}(\omega), \boldsymbol{k}(r, \omega))$ and we define $\boldsymbol{\vartheta}(r, \omega)=\left(\nabla_{\boldsymbol{L}} \boldsymbol{v}(r, \omega)\right)^{\perp}$. We denote with $\nabla$ the (covariant) directional derivative of a vector field. The use of the generalised line direction $\boldsymbol{L}$ instead of just the spatial line direction $\boldsymbol{l}$ here is necessary to capture the continuation of the dislocation lines on $S M$ correctly. We furthermore note that the above given definition of $\boldsymbol{\vartheta}$, which we motivate here from considerations on a single dislocation, turns out to be necessary for a consistent continuum formulation as will be explained in section 6.1 with reference to equation (13).

With the generalised velocity at hand, the evolution equation for the dislocation density measure defined in (6) is given by

$$
\partial_{t} \boldsymbol{\alpha}_{\mathrm{d}}=-\mathcal{L}_{\boldsymbol{V}} \boldsymbol{\alpha}_{\mathrm{d}}
$$

It should be noted that the concepts presented in the present section heavily rely on the formulation in terms of differential forms. While this is already 
important for the precise definition of $\boldsymbol{\alpha}_{\mathrm{d}}$, it is essential for the formulation of the evolution equation. The latter could in general not be formulated in terms of the curl-operator which is only defined in three (or two) dimensions. Furthermore, the concepts of differential forms and currents are essential to understand the relation between the motion of the single curve and the appearance of the Lie derivative in the evolution equation for the density measure, as discussed in more detail in [6].

\subsection{Geometrical restrictions}

We briefly discuss the question which dislocation states can be faithfully represented by the generalised measure defined in this section. Similar to the classical case where all dislocations passing through a certain volume element must have the same direction and orientation, we now have to assume that nearby dislocations with nearly the same direction and orientation (i.e. those which are passing the same volume element in $S M=M \times S^{2}$ ) have the same curvature. This restriction is obviously much weaker than the classical one, even though it is not easily visualised what this means for admissible distributions globally. Locally, however, there are several arguments which make this assumption seem not too restrictive. In a quasistatic picture the dislocation curvature will essentially be a function of the local stress, as the corresponding force on the dislocation would have to be compensated by the line-tension force which is proportional to the curvature. It should be noted that in a coarse grained theory, both the stress and the curvature have to be considered as coarse-grained quantities which still allow for fluctuations on the microscale e.g. due to the nearby dislocations. In a truely dynamic situation the above restriction will in general not be fulfilled. But for this case another advantage compared to the classical dislocation density tensor is striking. Even if one starts out from a more general dislocation distribution than can be reflected by the measure (i.e. from a distribution where different dislocations passing the same volume element in $S M$ have different curvatures), an averaged curvature is still a meaningful quantity which is not the case for the averaged line direction in the classical case. A simple indication for this is that a dislocation arrangement may have a zero net tangent vector such that the average orientation becomes meaningless; a zero average curvature in contrast still has a well defined meaning. Furthermore we consider the correspondence between averaged curvature and actual curvature not as essential as the one between averaged direction and actual line-direction; the latter is indispensable to be able to define a meaningful velocity field, whereas it may be feasible to replace the actual curvature by the averaged one.

\subsection{Relation to the classical dislocation density tensor}

Obviously it should be possible to recover the classical dislocation density and its evolution from the generalised dislocation density measure. To this end we introduce a concept which was already implicitly used in the preceding. Each tangent vector to $S M$ can be split into a spatial part and a rotatory part, as seen for example from $\boldsymbol{L}=(\boldsymbol{l}, \boldsymbol{k})$ and $\boldsymbol{V}=(\boldsymbol{v}, \boldsymbol{\vartheta})$. Similarly, each tangent vector $\boldsymbol{x}$ to the crystal manifold $M$ may be formally 'lifted' to obtain a tangent vector $\boldsymbol{x}^{\mathrm{h}}$ to $S M$ by setting $\boldsymbol{x}^{\mathrm{h}}=(\boldsymbol{x}, 0)$. With the aid of this concept we define the 
dislocation density tensor from the generalised dislocation density measure by its action on two tangent vectors $\boldsymbol{x}$ and $\boldsymbol{y}$ (tangent to a fixed point $r$ which is suppressed in the notation) as

$$
\boldsymbol{\alpha}(\boldsymbol{x}, \boldsymbol{y}):=\int_{S^{2}} \mathrm{i}_{\boldsymbol{y}^{\mathrm{h}}} \mathrm{i}_{\boldsymbol{x}^{\mathrm{h}}} \boldsymbol{\alpha}_{\mathrm{d}}=\int_{S^{2}} \rho(\omega) \operatorname{det}[\boldsymbol{l}(\omega), \boldsymbol{x}, \boldsymbol{y}] d \Omega(\omega) \otimes \boldsymbol{b} .
$$

This obviously defines a 2-form because it is antisymmetric in $\boldsymbol{x}$ and $\boldsymbol{y}$ and linear in both arguments because the same holds true for $\boldsymbol{\alpha}_{\mathrm{d}}$. Before we justify this definition by demonstrating that it yields the correct relation between the dislocation density tensor and the plastic distortion, we at first check that $\boldsymbol{\alpha}$ indeed defines a closed differential form. It will be shown that this is a direct consequence of the requirement that $\boldsymbol{\alpha}_{\mathrm{d}}$ is closed $\left(d \boldsymbol{\alpha}_{\mathrm{d}}=0\right)$. We demonstrate this in integral form and do refer to Appendix A for more on the integration of differential forms and the generalised Stokes' theorem. Let $\mathcal{G}$ be an open subset of the crystal which allows to apply Stokes theorem. Then we find

$$
\int_{\mathcal{G}} d \boldsymbol{\alpha} \stackrel{\text { Stokes }}{=} \int_{\partial \mathcal{G}} \boldsymbol{\alpha} \stackrel{(8)}{=} \int_{\partial \mathcal{G} \times S^{2}} \boldsymbol{\alpha}_{\mathrm{d}}^{\stackrel{\text { Stokes }}{\partial S^{2}=\emptyset}} \int_{\mathcal{G} \times S^{2}} d \boldsymbol{\alpha}_{\mathrm{d}}=0,
$$

which is equivalent to $d \boldsymbol{\alpha}=0$ because it must hold for every admissible $\mathcal{G}$.

To justify the definition of $\boldsymbol{\alpha}$ we first define the 1-form of the plastic distortion rate by

$$
\partial_{t} \boldsymbol{\beta}^{\mathrm{pl}}(\boldsymbol{x})=-\int_{S^{2}} \mathrm{i}_{\boldsymbol{x}^{\mathrm{h}}} \mathrm{i}_{\boldsymbol{V}} \boldsymbol{\alpha}_{\mathrm{d}}=-\int_{S^{2}} \rho(\omega) \operatorname{det}[\boldsymbol{l}(\omega), \boldsymbol{v}(\omega), \boldsymbol{x}] d \Omega(\omega) \otimes \boldsymbol{b} .
$$

That this definition recovers the classical definition of $\partial_{t} \boldsymbol{\beta}^{\mathrm{pl}}$ can be seen as follows. If the dislocations are moving in a plane one finds $\boldsymbol{l}(\omega) \times \boldsymbol{v}(\omega) \equiv$ $\pm v(\omega) \boldsymbol{n}$ with the glide plane normal $\boldsymbol{n}$ and a signed scalar velocity $v(\omega)$. The actual sign on the right hand side is a matter of convention for the scalar velocity $v$ (or optional for the normal $\boldsymbol{n}$ ) which we choose such that the minus sign holds. As a consequence we find $\operatorname{det}[\boldsymbol{l}(\omega), \boldsymbol{v}(\omega), \boldsymbol{x}]=(\boldsymbol{l}(\omega) \times \boldsymbol{v}(\omega)) \cdot \boldsymbol{x} \equiv-\boldsymbol{v} \boldsymbol{n} \cdot \boldsymbol{x}$. For a fixed glide system we may therefore, in accordance to the classical case (see section 2.1), characterise the plastic distortion rate by the dual 1-form $\boldsymbol{n}^{b}$ to the slip plane normal $\boldsymbol{n}$ as

$$
\partial_{t} \boldsymbol{\beta}^{\mathrm{pl}}:=\int_{S^{2}} \rho(\omega) v(\omega) d \Omega(\omega) \boldsymbol{n}^{\mathrm{b}} \otimes \boldsymbol{b} .
$$

This obviously recovers the classical definition via the Orowan equation. In the limit where only one orientation is present locally (i.e. , the dislocation lines form parallel bundles) this expression reduces to the one discussed previously in section 2.1 .

We now ask whether the above definition of the classical dislocation density tensor coincides with the classical relation between plastic distortion and plastic deformation, $\boldsymbol{\alpha}=d \boldsymbol{\beta}^{\mathrm{pl}}$. In fact we can only check this equation in rate form $\partial_{t} \boldsymbol{\alpha}=d \partial_{t} \boldsymbol{\beta}^{\mathrm{pl}}$. We consider a surface $\mathcal{F}$ in the crystal which allows to apply Stokes theorem and find

$\partial_{t} \int_{\mathcal{F}} \boldsymbol{\alpha} \stackrel{(8)}{=} \partial_{t} \int_{\mathcal{F} \times S^{2}} \boldsymbol{\alpha}_{\mathrm{d}} \stackrel{(7)}{=}-\int_{\mathcal{F} \times S^{2}} d \mathrm{i}_{\boldsymbol{V}} \boldsymbol{\alpha}_{\mathrm{d}} \underset{\partial S^{2}=\emptyset}{\text { Stokes }}-\int_{\partial \mathcal{F} \times S^{2}} \mathrm{i}_{\boldsymbol{V}} \boldsymbol{\alpha}_{\mathrm{d}} \stackrel{(9)}{=} \int_{\partial \mathcal{F}} \partial_{t} \boldsymbol{\beta}^{\mathrm{pl}}$. 
Because this holds for every admissible $\mathcal{F}$ this constitutes the integral version of the rate equation in question.

Obviously the curvature $k$ does not appear in the definitions of the classical objects. One may therefore ask whether or why the curvature is needed to predict the evolution of the system. The reason will become appearant in section 6 where we find the evolution of $\rho$ to be dependend on the curvature.

We summarise that from the generalised dislocation density measure $\boldsymbol{\alpha}_{\mathrm{d}}$ and its evolution equation the classical dislocation density theory including the plastic deformation can be recovered. The evolution of the plastic deformation is thereby directly deduced from the evolution of the dislocation state even in a fully three-dimensional formulation.

\section{The conservation laws}

The evolution equations both for the classical and for the generalised dislocation density measure can be interpreted as conservation laws. For the classical dislocation density the integral version of the evolution equation is determined by integration over a surface $\mathcal{F}$ as

$$
\partial_{t} \int_{\mathcal{F}} \boldsymbol{\alpha}=-\int_{\partial \mathcal{F}} \mathrm{i}_{\boldsymbol{v}} \boldsymbol{\alpha}=\int_{\partial \mathcal{F}} \partial_{t} \boldsymbol{\beta}^{\mathrm{pl}}
$$

This means that any change of the net Burgers vector content of $\mathcal{F}$ may only be due to the flow of net Burgers vector across the boundary $\partial \mathcal{F}$ of the surface. The 1 -form $\mathrm{i}_{\boldsymbol{v}} \boldsymbol{\alpha}$ may be viewed as the dislocation flux corresponding to a density of infinitesimal swept surfaces. We remind that in classical vector notation this 1 -form would in accordance with $\boldsymbol{\alpha}=\rho \mathrm{i}_{\boldsymbol{l}} d V$ be written as $\rho \boldsymbol{v} \times \boldsymbol{l}$, which makes the interpretation of $\boldsymbol{v} \times \boldsymbol{l}$ as infinitesimal swept surface obvious.

The corresponding conservation law for the generalised measure is obtained via integrating over a so-called hypersurface $\mathfrak{F}$ in the configuration space $S M$ :

$$
\partial_{t} \int_{\mathfrak{F}} \boldsymbol{\alpha}_{\mathrm{d}}=-\int_{\partial \mathfrak{F}} \mathrm{i}_{\boldsymbol{V}} \boldsymbol{\alpha}_{\mathrm{d}}
$$

A hypersurface is a subspace of co-dimension one, which means it has one dimension less than the surrounding space. In our case, $S M$ will for a threedimensional crystal have five dimensions (three spatial and two directional) and therefore $\mathfrak{F}$ is supposed to be a smooth four-dimensional subspace. The interpretation of the generalised conservation law is analogous to the classical one, although our visualisation struggles with the extra dimensions. But a special case was already treated at the end of the last subsection. There, the hypersurface $\mathfrak{F}$ arose as a product of a spatial surface $\mathcal{F}$ with the full directional space $S^{2}$, thus $\mathfrak{F}=\mathcal{F} \times S^{2}$. For this case the generalised conservation law (11) reproduces the classical one, as was seen in the calculation (10).

As a concluding remark we note that both conservation laws show that the conserved quantity in either case is the total number of dislocation loops and not the total line length. 


\section{Mean field approximation}

We already saw that the generalised deterministic dislocation density measure allows for a closed theory of plasticity in the sense that the evolution of the dislocation state uniquely determines the plastic deformation of the crystal. However, until this point we only gave a purely kinematic picture and assumed the velocity distribution which is needed to deduce the evolution of the density measure as given. A physically sound derivation of the dislocation velocity as a functional of the dislocation density measure, as it was obtained in [15] and [1] by statistical averaging for simplified two-dimensional systems, will be a challenging future task in three dimensions; but the first step towards a physically based modelling, namely the mean field approximation, can also easily be determined for the generalised measure. This simplest statistical approximation neglects the spatial correlations of the dislocations and assumes that the probability $\rho(r, \omega)$ to find a dislocation with line direction $\boldsymbol{l}(\omega)$ at the point $r$ does not depend on the arrangement of the surrounding dislocations (local density approximation). This implies that short-range interactions between the dislocations are neglected while long range interactions are still described correctly. One particular kind of short range interaction, namely the short range self-interaction of a dislocation, can however easily be included into the theory by using a line tension approximation.

\subsection{Internal stresses}

Long range interactions of dislocations are a topic of the classical continuum theory of dislocations and were extensively discussed by Kröner in [2] and [11]. There it is shown how the internal stress $\sigma_{\text {int }}(\boldsymbol{\alpha})$ can be determined from the classical dislocation density tensor $\boldsymbol{\alpha}$. As was shown in subsection 3.2 the classical dislocation density tensor $\boldsymbol{\alpha}$ can be determined from $\boldsymbol{\alpha}_{\mathrm{d}}$. Therefore the internal stress can be determined from the current dislocation state characterised by $\boldsymbol{\alpha}_{\mathrm{d}}$ by simple recourse to the classical theory. If additionally an external stress $\sigma_{\text {ext }}$ is applied, both stresses add up to $\sigma=\sigma_{\text {ext }}+\sigma_{\text {int }}$.

This allows for a simple self-consistent model when for example overdamped conservative dislocation motion is assumed. Consequently the dislocation velocity is taken to be proportional to the projection of the Peach-Koehler force $F_{\mathrm{PK}}=\sigma \cdot \boldsymbol{b} \times \boldsymbol{l}$ onto the glide plane. The mobility of the dislocations is taken to be the inverse of the so-called drag coefficient $B(\boldsymbol{l})$ which will in general depend on the line direction $\boldsymbol{l}$ explicitly. If we denote the projection operator of the glide plane as $P$ we thus find

$$
\boldsymbol{v}(\boldsymbol{l})=B^{-1}(\boldsymbol{l}) P\left(F_{\mathrm{PK}}\right)=B^{-1}(\boldsymbol{l}) P\left(\left(\sigma_{\mathrm{ext}}+\sigma_{\mathrm{int}}\right) \cdot \boldsymbol{b} \times \boldsymbol{l}\right) .
$$

\subsection{Line tension}

The line tension $T$ is formally defined as the change of stored energy due to an infinitesimal increase in length of the dislocation. For dislocations in contrast to taut elastic strings this concept may imply several subtleties; for example, the line-tension may be depend on the character of the dislocation and also, though weakly (logarithmically), on the arrangement of surrounding dislocations. Within the present qualitative discussion we avoid to specify the exact 
form of the line-tension. For us, the important characteristic of the line-tension approximation is that it produces a force $F_{\mathrm{t}}$ on the dislocation. This force locally points in the direction opposing the direction of maximal possible increase in line length when the dislocation moves. This means that it points in the direction of the curvature vector, thus $F_{\mathrm{t}}=T \boldsymbol{k}$. Consequently the dislocation velocity would be modified as

$$
\boldsymbol{v}(\boldsymbol{l})=B^{-1}(\boldsymbol{l}) P\left(\left(\sigma_{\mathrm{ext}}+\sigma_{\mathrm{int}}\right) \cdot \boldsymbol{b} \times \boldsymbol{l}+T \boldsymbol{k}\right) .
$$

\section{Explicit formulation in two dimensions}

The following example is a generalisation of the two-dimensional case treated by Sedláček et al. in [12], which was sketched at the end of section 2.1. Accordingly, we assume a system that is homogenous in the $z$ direction such that the evolution of the dislocation system is completely specified by their motion in the $x$ - $y$-plane. Different from Sedláček et. al. however, we allow for dislocations with different directions at each point. (This obviously implies that our volume element is large enough to contain dislocations moving on different glide planes such that the dislocations do not necessarily intersect each other.) We parametrise the directional space at each point by an angle $\varphi$ and therefore work on the configuration space $S M=\mathbb{R}^{2} \times S^{1}=\mathbb{R}^{2} \times[0,2 \pi)$. Please note that the angle $\varphi$, as a constituent of the configuration space, plays in this case a very different role than in the notion of Sedláček et al. [12] (see end of section 2.1 ), where it was considered as field-variable. A role similar to that of the angle $\varphi$ in [12] will, however, be taken by the (pseudo) scalar field-variable $k$ which represents the curvature.

We have to make a sign convention in order to define the generalised linedirection $\boldsymbol{L}$. In general this takes the form

$$
\boldsymbol{L}=\cos \varphi \partial_{x}+\sin \varphi \partial_{y}+k \partial_{\varphi}
$$

with a (pseudo) scalar field $k$. The sign of $k$ is a matter of convention and we consider a circular loop oriented counter-clockwise as positively curved, $k>0$.

The definition of $\boldsymbol{\alpha}_{\mathrm{d}}$ via interior multiplication necessitates the choice of a volume element $d \mathcal{V}$ on $S M$. We chose to work with the volume element $d \mathcal{V}=d x \wedge d y \wedge d \varphi$, which facilitates the calculations. Every other choice of the volume element would yield an equivalent theory if $\rho$ would be adjusted accordingly. With the current choice the dislocation density measure reads

$$
\boldsymbol{\alpha}_{\mathrm{d}}=\rho \mathrm{i}_{\boldsymbol{L}} d \mathcal{V}=\rho(\cos \varphi d y \wedge d \varphi-\sin \varphi d x \wedge d \varphi+k d x \wedge d y) \otimes \boldsymbol{b}
$$

For later use we introduce a vector field $\boldsymbol{R}=\rho \boldsymbol{L}$ which reads

$$
\boldsymbol{R}=\rho \cos \varphi \partial_{x}+\rho \sin \varphi \partial_{y}+\rho k \partial_{\varphi}=: R^{x} \partial_{x}+R^{y} \partial_{y}+R^{\varphi} \partial_{\varphi}
$$

We can recapture the field variables $\rho$ and $k$ which characterise the dislocation state from this vector field as

$$
\rho=\sqrt{\left(R^{x}\right)^{2}+\left(R^{y}\right)^{2}} \quad \text { and } \quad k=\left(\frac{R^{\varphi}}{\rho}\right) .
$$


The continuity equation $d \boldsymbol{\alpha}_{\mathrm{d}}=0$ is equivalent to the requirement that $\operatorname{Div} \boldsymbol{R}=$ 0 , because $\boldsymbol{\alpha}_{\mathrm{d}}=\mathrm{i}_{\boldsymbol{R}} d \mathcal{V} \otimes \boldsymbol{b}$. For the current example we find

$$
d \boldsymbol{\alpha}_{\mathrm{d}}=\left\{\left(l^{x} \partial_{x}+l^{y} \partial_{y}+k \partial_{\varphi}\right) \rho+\rho \partial_{\varphi} k\right\} d \mathcal{D} \otimes \boldsymbol{b}=0,
$$

as the necessary coupling between $\rho$ and $k$.

\subsection{Kinematic evolution equation}

The evolution of the above defined measure can clearly be described through the evolution of $\rho$ and $k$. In order to deduce the corresponding equations we first analyse the generalised velocity for which we make a further sign convention. We will regard the velocity of a positively oriented loop as positive if the loop expands. This fixes the sign of the pseudo scalar velocity $v$ in the representation of the vectorial velocity as $\boldsymbol{v}(\varphi)=v \sin \varphi \partial_{x}-v \cos \varphi \partial_{y}$. The rotation velocity, in general given by $\boldsymbol{\vartheta}=\left(\nabla_{\boldsymbol{L}} \boldsymbol{v}\right)^{\perp}$, is then found as

$$
\begin{aligned}
\boldsymbol{\vartheta} & =\left(\boldsymbol{L}\left(v \sin \varphi \partial_{x}-v \cos \varphi \partial_{y}\right)\right)^{\perp} \\
& =\left(\boldsymbol{L}(v) \sin \varphi \partial_{x}+v k \cos \varphi \partial_{x}-\boldsymbol{L}(v) \cos \varphi \partial_{y}+v k \sin \varphi \partial_{y}\right)^{\perp} \\
& =-\boldsymbol{L}(v)\left(-\sin \varphi \partial_{x}+\cos \varphi \partial_{y}\right) \\
& =-\boldsymbol{L}(v) \partial_{\varphi}=: \vartheta \partial_{\varphi} .
\end{aligned}
$$

Therefore the generalised velocity reads

$$
\boldsymbol{V}=v \sin \varphi \partial_{x}-v \cos \varphi \partial_{y}-\boldsymbol{L}(v) \partial_{\varphi} .
$$

For the further analysis we recall a general fact resulting from the product rules for the Lie-derivative given in Appendix A. They yield

$$
\begin{aligned}
\partial_{t} \boldsymbol{\alpha}_{\mathrm{d}} & =-\mathcal{L}_{\boldsymbol{V}} \boldsymbol{\alpha}_{\mathrm{d}} \\
& =\left\{-\boldsymbol{V}(\rho) \mathrm{i}_{\boldsymbol{L}} d \mathcal{V}-\rho \mathrm{i}_{[\boldsymbol{V}, \boldsymbol{L}]} d \mathcal{V}-\rho \operatorname{Div}(\boldsymbol{V}) \mathrm{i}_{\boldsymbol{L}} d \mathcal{V}\right\} \otimes \boldsymbol{b} \\
& =\left\{-\operatorname{Div}(\rho \boldsymbol{V}) \mathrm{i}_{\boldsymbol{L}} d \mathcal{V}-\rho \mathrm{i}_{[\boldsymbol{V}, \boldsymbol{L}]} d \mathcal{V}\right\} \otimes \boldsymbol{b} .
\end{aligned}
$$

The Lie-bracket appearing on the right hand side of the last equation is explicitely calculated as

$$
\begin{aligned}
{[\boldsymbol{V}, \boldsymbol{L}]=} & (\boldsymbol{V}(\cos \varphi)-\boldsymbol{L}(v \sin \varphi)) \partial_{x} \\
& +(\boldsymbol{V}(\sin \varphi)+\boldsymbol{L}(v \cos \varphi)) \partial_{y}+(\boldsymbol{V}(k)-\boldsymbol{L}(\vartheta)) \partial_{\varphi} \\
= & (-\vartheta \sin \varphi-\boldsymbol{L}(v) \sin \varphi-v k \cos \varphi) \partial_{x} \\
& \quad+(\vartheta \cos \varphi+\boldsymbol{L}(v) \cos \varphi-v k \sin \varphi) \partial_{y}+(\boldsymbol{V}(k)-\boldsymbol{L}(\vartheta)) \partial_{\varphi} \\
= & -v k \cos \varphi \partial_{x}-v k \sin \varphi \partial_{y}+(\boldsymbol{V}(k)-\boldsymbol{L}(\vartheta)) \partial_{\varphi} .
\end{aligned}
$$

This result is used to obtain the evolution equation of the vector-field $\boldsymbol{R}=\rho \boldsymbol{L}$ using the fact that $\partial_{t} \boldsymbol{\alpha}_{\mathrm{d}}=\mathrm{i}_{\partial_{t} \boldsymbol{R}} d \boldsymbol{V} \otimes \boldsymbol{b}$. By inserting equation (13) into (12) we therefore find

$$
\begin{aligned}
\partial_{t} \boldsymbol{R}=(-\operatorname{Div}(\rho \boldsymbol{V})+\rho v k) \cos \varphi \partial_{x}+ & (-\operatorname{Div}(\rho \boldsymbol{V})+\rho v k) \sin \varphi \partial_{y} \\
& +(-\operatorname{Div}(\rho \boldsymbol{V}) k-\rho(\boldsymbol{V}(k)-\boldsymbol{L}(\vartheta))) \partial_{\varphi} .
\end{aligned}
$$


It should be noted that the spatial part of $\boldsymbol{R}$ stays a multiple of the canonical direction $\boldsymbol{l}(\varphi)=\cos \varphi \partial_{x}+\sin \varphi \partial_{y}$, and that this results from the definition $\vartheta=-\boldsymbol{L}(v)$, as can be seen from the derivation of equation (13). In fact this is the reason why the definition of $\boldsymbol{\vartheta}$ as given in section 3 is a necessary condition for the evolution equation to be consistent with the definition of $\boldsymbol{\alpha}_{\mathrm{d}}$ in equation (6).

From equation (14) we easily obtain the evolution of the density as

$$
\partial_{t} \rho=\partial_{t} \sqrt{\left(R^{x}\right)^{2}+\left(R^{y}\right)^{2}}=-\operatorname{Div}(\rho \boldsymbol{V})+\rho v k,
$$

and the evolution of the curvature as

$$
\begin{aligned}
\partial_{t} k & =\partial_{t}\left(\frac{R^{\varphi}}{\rho}\right)=\frac{\partial_{t} R^{\varphi} \rho-R^{\varphi} \partial_{t} \rho}{\rho^{2}} \\
& =\frac{\rho(-\operatorname{Div}(\rho \boldsymbol{V}) k-\rho(\boldsymbol{V}(k)-\boldsymbol{L}(\vartheta)))-\rho k(-\operatorname{Div}(\rho \boldsymbol{V})+\rho v k)}{\rho^{2}} \\
& =-v k^{2}+\boldsymbol{L}(\vartheta)-\boldsymbol{V}(k) .
\end{aligned}
$$

We emphasise the close formal analogy with the evolution equations found by Sedláček et al. as given in equation (5). The evolution equation of the density function (15) is formally analogous to the result cited above (note, however, that the Div-operator is operating on a higher-dimensional space which includes the orientation), and the role of the curvature for the increase in total line length is the same. The correct evolution of the curvature therefore is essential and the corresponding equation (16) shall be analysed in more detail. The first two terms in the last line are easily seen to represent the Lagrangian time-derivative of the curvature. The first term, $-v k^{2}$, is the change of curvature like found for a circular loop of radius $r=1 / k$, which expands (or shrinks) with velocity $v$. In this case we have $r(t)=r+v t$ and therefore

$$
\partial_{t} k=\partial_{t}\left(\frac{1}{r}\right)=\frac{-v}{r^{2}}=-v k^{2} .
$$

The second term, $\boldsymbol{L}(\vartheta)=-\boldsymbol{L}(\boldsymbol{L}(v))$, accounts for the second derivative of the velocity along the dislocation line, which clearly yields a proportional change in curvature. The third term, $-\boldsymbol{V}(k)$, accounts for changes of the curvature in the direction of motion.

We conclude the explicit calculations by deducing the classical dislocation density tensor and the plastic deformation rate for this case. We assume a spatial vector $\boldsymbol{x}=\mathrm{x}^{x} \partial_{x}+\mathrm{x}^{y} \partial_{y}$ as given. This is canonically lifted to the configuration space as $\boldsymbol{x}^{\mathrm{h}}=(\boldsymbol{x}, 0):=\mathrm{x}^{x} \partial_{x}+\mathrm{x}^{y} \partial_{y}+0 \partial_{\varphi}$. The interior multiplication of $\boldsymbol{\alpha}_{\mathrm{d}}$ with $\boldsymbol{x}^{\mathrm{h}}$ is found as

$$
\mathrm{i}_{\boldsymbol{x}^{\mathrm{h}}} \boldsymbol{\alpha}_{\mathrm{d}}=\{-\rho k \mathrm{x}^{y} d x+\rho k \mathrm{x}^{x} d y+\rho \underbrace{\left(\mathrm{x}^{y} \cos \varphi-\mathrm{x}^{x} \sin \varphi\right)}_{=\operatorname{det}[\boldsymbol{l}, \boldsymbol{x}]} d \varphi\} \otimes \boldsymbol{b} .
$$

Therefore holds $\mathrm{i}_{\boldsymbol{x}^{\mathrm{h}}} \boldsymbol{\alpha}_{\mathrm{d}}\left(\partial_{\varphi}\right)=\rho(\varphi) \operatorname{det}[\boldsymbol{l}(\varphi), \boldsymbol{x}] \boldsymbol{b}$ and we obtain for the classical dislocation density tensor in analogy to $(8)$ :

$$
\boldsymbol{\alpha}(\boldsymbol{x})=\int_{S^{1}} \mathrm{i}_{\boldsymbol{x}^{\mathrm{h}}} \boldsymbol{\alpha}_{\mathrm{d}} \otimes \boldsymbol{b}=\int_{0}^{2 \pi} \rho(\varphi) \operatorname{det}[\boldsymbol{l}(\varphi), \boldsymbol{x}] d \varphi \otimes \boldsymbol{b}
$$


Accordingly we have $\boldsymbol{\alpha}_{x}=\boldsymbol{\alpha}\left(\partial_{x}\right)=-\int_{0}^{2 \pi} \rho \sin \varphi d \varphi \boldsymbol{b}$ and $\boldsymbol{\alpha}_{y}=\boldsymbol{\alpha}\left(\partial_{y}\right)=$ $\int_{0}^{2 \pi} \rho \cos \varphi d \varphi \boldsymbol{b}$. If in turn we want to represent $\boldsymbol{\alpha}$ by a density $\bar{\rho}$ and a linedirection $\overline{\boldsymbol{l}}$ as $\boldsymbol{\alpha}=\bar{\rho} \mathrm{i} \overline{\boldsymbol{l}} d V \otimes \boldsymbol{b}$ we find $\bar{\rho}=\left(\boldsymbol{\alpha}_{x}^{2}+\boldsymbol{\alpha}_{y}^{2}\right)^{1 / 2}$ and $\overline{\boldsymbol{l}}=\bar{\rho}^{-1}\left(\boldsymbol{\alpha}_{y} \partial_{x}-\boldsymbol{\alpha}_{x} \partial_{y}\right)$.

Analogously and in accordance with (9) we define the plastic deformation rate via

$$
\begin{aligned}
\partial_{t} \boldsymbol{\beta}^{\mathrm{pl}} & =-\int_{S^{1}} \mathrm{i}_{\boldsymbol{V}} \boldsymbol{\alpha}_{\mathrm{d}}=-\int_{0}^{2 \pi} \mathrm{i}_{\boldsymbol{V}} \boldsymbol{\alpha}_{\mathrm{d}}\left(\partial_{\varphi}\right) d \varphi \\
& =-\int_{0}^{2 \pi} \rho(\varphi) \operatorname{det}[\boldsymbol{l}(\varphi), \boldsymbol{v}(\varphi)] d \varphi \boldsymbol{b} \\
& =\int_{0}^{2 \pi} \rho(\varphi) v(\varphi) d \varphi \boldsymbol{b} .
\end{aligned}
$$

We again note that this is the common definition of the plastic distortion rate as determined from Orowan's equation.

\subsection{Example}

A very simple example which is not accessible to the other dislocation density measures mentioned in this paper is obtained from assuming an homogeneous distribution of circular dislocation loops with fixed radius $r$ as initial state. If $a$ denotes the average spacing between the centres of these loops, the density function is constant on the configuration space and given by $\rho=r / a^{2}$ within the two-dimensional model introduced above. Let furthermore the velocity $\boldsymbol{v}$ be such that the scalar velocity $v$ is constant and consequently $r(t)=r+v t$. Considering that $k=1 / r$ the evolution equations then reduce to $\partial_{t} \rho=\rho v k=$ $v / a^{2}$ and $\partial_{t} k=-v k^{2}=-v / r^{2}$. This obviously complies with what is obtained when the time dependent radius is inserted directly to the definition of $\rho$ and $k$.

In this example the Kröner tensor vanishes identically and the plastic distortion rate is given by $\partial_{t} \boldsymbol{\beta}=2 \pi \rho v \boldsymbol{b}$.

\section{Summary and Outlook}

We defined a dislocation density measure as a differential form on the space made up of all directions at each point of a crystal. It was shown that this measure and its evolution equation are in every respect natural generalisations of the classical dislocation density and its evolution. The evolution of the generalised dislocation density measure is able to reflect in a kinematically precise fashion the evolution of a dislocation system where dislocations passing the same volume element in $S M$ have the same curvature. The plastic distortion rate and its relation to the classical dislocation density tensor can be recovered from the generalised evolution equation in a mathematically consistent and rigorous manner. As a first step towards a physically based three-dimensional theory of plasticity we presented a mean-field theory of curved dislocations built upon the introduced dislocation density measure. In a two-dimensional example the abstract notions were animated and the curvature of the dislocations was shown to play a crucial role for the correct evolution of the dislocation density.

The presented approach includes several restrictions and simplifying assumptions: The geometrical restriction on faithfully representable dislocation distri- 
butions was already mentioned in the last paragraph. A proper understanding of the consequences of this restriction will have to be addressed in future work. No dislocation sources or annihilation are included into the model. The inherent change of total line length due to bowing of dislocations partly compensates for these effects - note, for instance, that the annihilation of two dislocation segments can be interpreted as the formation and subsequent motion of a segment with initially infinite negative curvature upon encounter of two loops. Furthermore the approach was restricted to one active glide system. From a mathematical point of view the extension to several glide systems does not introduce new fundamental problems but would require to work with several similar dislocation density measures. From a physical viewpoint, however, the treatment of the mutual interactions between the dislocations on different glide systems is likely to complicate things a lot. Mathematical ideas for the modelling of some of these interactions have been presented by El-Azab in [4].

For a numerical implementation of the present theory the extra dimensions from the directional space together with the inherent non-locality form a major challenge. Otherwise the obtained evolution equations together with the Orowan equation may substitute the constitutive rules used to determine the plastic deformation in usual Finite Element schemes in the same fashion like discrete dislocation simulations were used in the so-called discrete-continuum simulations by Lemarchand et al. [16]. We remark that with this procedure the long-range internal stresses needed for a mean-field theory emerge directly from the elastic distortions which have to compensate for inhomogeneous plastic distortions. Simple numerical examples based on the presented approach can be found in [7].

\section{Acknowledgements}

We gratefully acknowledge partial financial support from the European Commission through the Marie Curie Research Training Network SizeDepEn under contract no. MRTN-CT-2003-504634.

\section{A Differential Forms}

Differential forms are a standard tool in differential topology and differential geometry as well as in different branches of physics. Accordingly there is a huge number of standard text-books dealing with differential forms. A good introduction is given in [17], whilst [18] serves as reference for the results shown and notations used in the following. We consistently use the Einstein summation convention.

\section{A.1 Vector fields and differential 1-forms}

Before we introduce differential forms we revisit some notations commonly used in differential geometry. An important concept is the understanding of tangent vectors $\left.\boldsymbol{x}\right|_{r}$ (vectors annexed to a spatial point $r$ ) as differential operators which act on functions $f$ at $r$ by $\left.\boldsymbol{x}\right|_{r}(f)=\left.\mathrm{x}^{i} \frac{\partial}{\partial x^{i}}\right|_{r}(f)$. The underlying space is considered $n$-dimensional and for the above notion it makes no difference whether we think of it as a general $n$-dimensional manifold in a chart or simply of $\mathbb{R}^{n}$ 
with standard co-ordinates. The $\left.\frac{\partial}{\partial x^{i}}\right|_{r}=:\left.\partial_{i}\right|_{r}$ can be considered as elements of a local basis for tangent vectors.

In addition to tangent vectors we define co-vectors or 1-forms (sometimes also called Pfaffian forms) as locally linear mappings from a tangent space to the real numbers. Each 1 -form $\boldsymbol{\omega}$ is locally given by $n$ functions $\omega_{i}(r)$ (similar to a vector) by setting $\omega_{i}(r)=\boldsymbol{\omega}\left(\left.\partial_{i}\right|_{r}\right)$. Formally we can define a local basis $\left.d x^{i}\right|_{r}$ for co-tangent vectors by demanding $\left.d x^{i}\right|_{r}\left(\left.\partial_{j}\right|_{r}\right)=\delta_{j}^{i}$, with the Kronecker delta $\delta_{j}^{i}$. Then obviously holds $\boldsymbol{\omega}=\omega_{i} d x^{i}$. Best known example of 1-forms are those arising as differentials of a function $f$ denoted by $d f=\partial_{i} f d x^{i}$. They act on a vector $\boldsymbol{x}$ in a dual way to the vector acting on the function $f$ by $d f(\boldsymbol{x})=\mathrm{x}^{i} \partial_{i} f$.

\section{A.2 Higher-order differential forms}

$\mathrm{A}$ (differential) $p$-form is a co-variant tensor $\mathbf{A}=A_{i_{1} \cdots i_{p}} d x^{i_{1}} \otimes \cdots \otimes d x^{i_{p}}$ of order $p$ which obeys for each permutation $\pi$ of order $p$ the relation $\mathbf{A}\left(\boldsymbol{x}_{\pi(1)}, \ldots, \boldsymbol{x}_{\pi(p)}\right)=$ $\operatorname{sign} \pi \mathbf{A}\left(\boldsymbol{x}_{1}, \ldots, \boldsymbol{x}_{p}\right)$. The integer $p$ is called the degree of the form; functions are considered as 0 -forms. We define the alternating 'wedge' product $\wedge$ of a $p$-form $\boldsymbol{\omega}$ and a $q$-form $\boldsymbol{\theta}$ as the $(p+q)$-form given by

$$
\boldsymbol{\omega} \wedge \boldsymbol{\theta}\left(\boldsymbol{x}_{1}, \ldots, \boldsymbol{x}_{p+q}\right)=\frac{1}{p ! q !} \sum_{\pi} \operatorname{sign} \pi \boldsymbol{\omega} \otimes \boldsymbol{\theta}\left(\boldsymbol{x}_{\pi(1)}, \ldots, \boldsymbol{x}_{\pi(p+q)}\right) .
$$

This product is associative, thus $(\boldsymbol{\omega} \wedge \boldsymbol{\theta}) \wedge \boldsymbol{\eta}=\boldsymbol{\omega} \wedge(\boldsymbol{\theta} \wedge \boldsymbol{\eta})$, and for $\boldsymbol{\omega}$ and $\boldsymbol{\theta}$ like in (17) holds $\boldsymbol{\omega} \wedge \boldsymbol{\theta}=(-1)^{p q} \boldsymbol{\theta} \wedge \boldsymbol{\omega}$. We receive a basis for the space of differential $p$-forms from the alternating products $d x^{i_{1}} \wedge \cdots \wedge d x^{i_{p}}$ of the basis of 1 -forms $d x^{i}$ by requiring the indices to be sorted by $i_{1}<\cdots<i_{p}$. Each $p$-form $\boldsymbol{\omega}$ can then be written as $\boldsymbol{\omega}=\omega_{i_{1} \cdots i_{p}} d x^{i_{1}} \wedge \cdots \wedge d x^{i_{p}}$.

For a $p$-form $\boldsymbol{\omega}$ the inner multiplication with $\boldsymbol{x}$ is the $(p-1)$-form $\mathrm{i}_{\boldsymbol{x}} \boldsymbol{\omega}$ defined by $\mathrm{i}_{\boldsymbol{x}} \boldsymbol{\omega}\left(\boldsymbol{x}_{1}, \ldots, \boldsymbol{x}_{p-1}\right)=\boldsymbol{\omega}\left(\boldsymbol{x}, \boldsymbol{x}_{1}, \ldots, \boldsymbol{x}_{p-1}\right)$. In standard tensor notation this is a contraction of $\boldsymbol{x}$ with the first index of $\boldsymbol{\omega}$.

\section{A.3 Calculus on differential forms}

The exterior derivative $d$ of a $p$-form $\boldsymbol{\omega}$ is a $(p+1)$-form defined by

$$
d \boldsymbol{\omega}=d \omega_{i_{1} \cdots i_{p}} \wedge d x^{i_{1}} \wedge \cdots \wedge d x^{i_{p}}=\frac{\partial \omega_{i_{1} \cdots i_{p}}}{\partial x^{i}} d x^{i} \wedge d x^{i_{1}} \wedge \cdots \wedge d x^{i_{p}} .
$$

In standard three-dimensional space $d$ corresponds to the grad-, curl- and divoperator on $0-$, 1- and 2-forms, respectively. As a simple consequence from standard calculus it is obtained $d \circ d \equiv 0$ (compare curl grad $\equiv 0$ and div curl $\equiv$ $0)$. Differential forms $\boldsymbol{\omega}$ with $d \boldsymbol{\omega}=0$ are called 'closed'.

Another important differential operation in the theory of differential forms is the so-called Lie-derivative. The Lie-derivative of a differential form in the direction of a vector-field $\boldsymbol{x}$ leaves the degree of a differential form fixed. It can be defined by combining the exterior derivative and the inner multiplication as

$$
\mathcal{L}_{\boldsymbol{x}} \boldsymbol{\omega}=d \mathrm{i}_{\boldsymbol{x}} \boldsymbol{\omega}+\mathrm{i}_{\boldsymbol{x}} d \boldsymbol{\omega}
$$

The Lie-derivative of differential forms emerges as generalisation of the Liederivative defined on vector fields, which is usually written with the so-called 
Lie-brackets $[\cdot, \cdot]$. In local co-ordinates the Lie-derivative of a vector field $\boldsymbol{y}$ in the direction of $\boldsymbol{x}$ reads

$$
\mathcal{L}_{\boldsymbol{x}}(\boldsymbol{y})=:[\boldsymbol{x}, \boldsymbol{y}]=\left(\mathrm{x}^{j} \partial_{j}\left(\mathrm{y}^{i}\right)-\mathrm{y}^{j} \partial_{j}\left(\mathrm{x}^{i}\right)\right) \partial_{i} .
$$

This allows to formulate the fact that

$$
\mathcal{L}_{\boldsymbol{x}} \mathrm{i}_{\boldsymbol{y}} \boldsymbol{\omega}=\mathrm{i}_{[\boldsymbol{x}, \boldsymbol{y}]} \boldsymbol{\omega}+\mathrm{i}_{\boldsymbol{y}} \mathcal{L}_{\boldsymbol{x}} \boldsymbol{\omega},
$$

for each $p$-form $\boldsymbol{\omega}$. Furthermore holds for every smooth function $f$ :

$$
\mathcal{L}_{\boldsymbol{x}}(f \boldsymbol{\omega})=\boldsymbol{x}(f) \boldsymbol{\omega}+f \mathcal{L}_{x} \boldsymbol{\omega}
$$

If a standard volume density $d V$ is defined on the underlying space (thus each $n$-form $\boldsymbol{\omega}$ can be written as $\boldsymbol{\omega}=\rho d V$ ), the divergence of a vector-field $\boldsymbol{x}$ with respect to this volume density is defined by

$$
\mathcal{L}_{\boldsymbol{x}} d V=d\left(\mathrm{i}_{\boldsymbol{x}} d V\right)=\operatorname{div} \boldsymbol{x} d V .
$$

\section{A.4 Integration of differential forms}

Maybe the most important property of differential $p$-forms is that they can be invariantly integrated over sub-spaces of dimension $p$. For reasons of space we shall only give four examples in standard three dimensional space. A 0 -form $f$ is 'integrated' over a point $r$ as $\int_{r} f=f(r)$; a 1-form $\varepsilon$ is integrated over a curve $c:[0, T) \rightarrow \mathbb{R}^{3} / t \mapsto c(t)$ via $\int_{c} \varepsilon=\left.\int_{0}^{T} \varepsilon\right|_{c(t)}\left(\frac{d c}{d t}\right) d t$; a 2 -form $\boldsymbol{\zeta}$ is integrated over a surface. Let $\mathcal{F}$ be a surface parameterised by a map $a: O \rightarrow \mathbb{R}^{3} /(x, y) \mapsto$ $\boldsymbol{a}(x, y)$, with an open subset $O \subset \mathbb{R}^{2}$, such that $\mathcal{F}=a(O)$. Then it is $\int_{\mathcal{F}} \boldsymbol{\zeta}=$ $\left.\iint_{O} \boldsymbol{\zeta}\right|_{\boldsymbol{a}(x, y)}\left(\frac{d \boldsymbol{a}}{d x}, \frac{d \boldsymbol{a}}{d y}\right) d x d y$; A 3 -form $\boldsymbol{\delta}$ is integrated over a suitable domain $\mathcal{G} \subset$ $\mathbb{R}^{3}$ as $\int_{\mathcal{G}} \boldsymbol{\delta}=\left.\iiint_{\mathcal{G}} \boldsymbol{\delta}\right|_{(x, y, z)}\left(\partial_{x}, \partial_{y}, \partial_{z}\right) d x d y d z$ if $\mathcal{G}$ is parametrised by standard co-ordinates. If we write $\boldsymbol{\delta}=\rho d V$ with a density function $\rho$ this yields the common form $\int_{\mathcal{G}} \boldsymbol{\delta}=\int_{\mathcal{G}} \rho d V$. The term 'invariantly' used above refers to the fact that the transformation law for differential forms automatically includes the change of variables formula as known for the integration of functions.

\section{A.5 Stokes Theorem}

The integral theorems of Gauss and Stokes turn out to be special cases of the general Stokes' theorem which says that for a differential $(n-1)$-form on a $n$-dimensional manifold $M$ with boundary $\partial M$ holds

$$
\int_{M} d \boldsymbol{\omega}=\int_{\partial M} \boldsymbol{\omega} .
$$

\section{References}

[1] I. Groma, F. F. Csikor, and M. Zaiser, Acta Mater. 511271 (2003).

[2] E. Kröner, Kontinuumstheorie der Versetzungen und Eigenspannungen. (Springer-Verlag, Berlin, 1958) 
[3] A. M. Kosevich, in Dislocations in Solids volume 1, edited by F. R. Nabarro (North Holland, Amsterdam, 1979) pp 33-142.

[4] A. El-Azab, Phys. Rev. B, 6111956 (2000).

[5] T. Hochrainer, submitted to J. Geom. and Phys. (2006).

[6] T. Hochrainer and M. Zaiser, in Proc. Internat Conf. on Statistical Mechanics of Plasticity and Related Instabilities PoS(SMPRI2005)002 (2006).

[7] M. Zaiser, N. Nikitas, T. Hochrainer and E. C. Aifantis, Phil. Mag. (this volume).

[8] J. F. Nye, Acta Metallurgica, 1(2) 153 (1953).

[9] K. Kondo, in Proc. 2. Japan nat. Congress of appl. Mech. (1952).

[10] B. A. Bilby, R. Bullough, and E. Smith. Proc. roy. Soc. London 231263 (1955).

[11] E. Kröner, Continuum theory of defects. (North Holland Publ. Comp., 1981).

[12] R. Sedláček, J. Kratochvil, and E. Werner, Phil. Mag. 833735 (2003).

[13] T. Mura. Phil. Mag. 8 (89) 843 (1963).

[14] M. Zaiser and T. Hochrainer, Scripta Mater. 54717 (2006).

[15] M. Zaiser, M. C. Miguel, and I. Groma, Phys. Rev. B 64224102 (2001).

[16] C. Lemarchand, B. Devincre, and L. P. Kubin. J. Mech. and Phys. Solids 491969 (2001).

[17] M. P. do Carmo. Differential Forms and Applications. (Springer-Verlag, Berlin-Heidelberg, 1994).

[18] F. W. Warner. Foundations of Differentiable Manifolds and Lie Groups. (Springer-Verlag, New York, 2nd ed. 1983). 
sophical Magazine \& Philosophieatgdagangine2LI

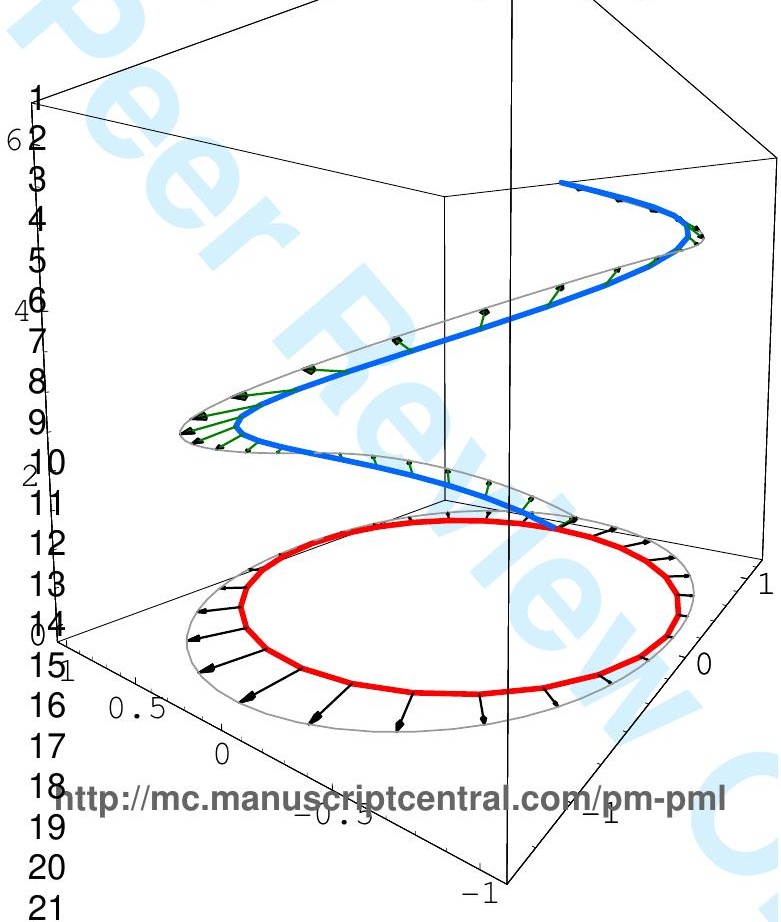

